\title{
Home Care for the Elderly under the Epidemic of Novel Coronavirus Pneumonia
}

\author{
Jieling Chen, Lin Wang*, Ruiying Huang, Jiali Chen \\ Transitional Care Department, the First Affiliated Hospital, Jinan University, Guangzhou, China \\ Email address: \\ chenjieling1356@126.com (Jieling Chen), Wanglin18903078509@126.com (Lin Wang), 165903802@qq.com (Ruiying Huang), \\ chenjialibella@163.com (Jiali Chen) \\ ${ }^{*}$ Corresponding author
}

\section{To cite this article:}

Jieling Chen, Lin Wang, Ruiying Huang, Jiali Chen. Home Care for the Elderly under the Epidemic of Novel Coronavirus Pneumonia. American Journal of Nursing Science. Vol. 9, No. 3, 2020, pp. 145-148. doi: 10.11648/j.ajns.20200903.22

Received: April 11, 2020; Accepted: April 27, 2020; Published: May 14, 2020

\begin{abstract}
Objective: To explore the effects of home care in the elderly patients under the epidemic of COVID-19. Methods: According to the hospital deployment and guidance documents, a series of actions were worked out, including the home care service process and contingency plan, training of the knowledge of novel coronavirus pneumonia (NCP), providing psychological intervention for nurses, providing home care for elderly patients at home and education about home prevention of COVID-19 for the elderly family. Results: From January 19 to March 31, 2020, the home visiting nurses provided home care service for 93 elderly patients at home, representing a $20 \%$ increase over the same period last year. The home care service included retention / replacement of gastric tube, retention / replacement of urinary tube, pressure ulcer care, sputum suction care, maintenance of peripherally inserted central catheters and venous blood collection for 127 times. The home visiting nurses were in good physical condition without any bad mood. Conclusion: Under the epidemic of COVID-19, more home-based elderly patients had home care service needs. Home care could not only take advantage under the epidemic situation, but also meet the medical needs of elderly patients at home, and effectively alleviate the burden of epidemic prevention and reduce the risk of cross infection. Home care could play a great role in prevention and control of the COVID-19 epidemic.
\end{abstract}

Keywords: Home Care, Novel Coronavirus Pneumonia, Epidemic

\section{Introduction}

Since December 2019, novel coronavirus (COVID-19) was identified in Wuhan, China [1]. The COVID-19 has rapidly spread to all over China and the world. It was found out that the COVID-19 can be transmitted from person to person through droplets, contact and aerosol [2]. It can cause symptoms including fever, cough, difficulty in breathing and invasive lesions on both lungs of the patients which has become a major public health issue [3]. On January 28, 2020, the notice on the prevention and control of novel coronavirus pneumonia (NCP) in the elderly issued by Health Secretary for the Elderly of National Health Commission of China [4] showed that most of the patients with severe NCP were elderly. The elderly will be in critical condition once they are infected due to various physiological degeneration or a variety of chronic diseases. Accordingly, the local governments need to do the health management of the elderly at home by the characteristics of home-based elderly people in various places. China is in the rapid development stage of population aging in recent years, the elderly population aged 60 years and above reached 250 million by the end of 2018 [5]. The elderly are the most important group suffering from chronic diseases. Studies showed that $80 \%$ of the elderly suffer from chronic diseases, and the average person suffers from two or three diseases, and each elderly person has two or three diseases on average [6]. Besides, the elderly have a long course of chronic diseases and they need continuous treatment and rehabilitation care [7]. The readmission rate of elderly patients with chronic diseases is high, which brings a heavy burden to hospitals and their families [8]. In a word, the elderly have a greater demand for medical, nursing and life care than others. It has become an important issue to meet the medical care needs of elderly patients at home under the epidemic of NCP that should not be 
ignored.

Home care refers to that nurses go to the home of patients to provide personalized and professional nursing service for patients with care needs, so as to achieve the goals of disease prevention, health promotion and health maintenance [9]. It can be used as a continuation and supplement of the inpatient treatment model. Our Transitional Care Department in the hospital have been providing home care service for the elderly patients at home for more than 10 years. The home visiting nurses still provided home care service for the elderly at home during the NCP epidemic and got good returns.

\section{Methods}

\subsection{Participants}

The elderly who were provided home care by home visiting nurses were selected in this study during the NCP epidemic period from January 19 to March 31, 2020.

\subsection{Procedure}

\subsubsection{Developing the Process of Home Care and Contingency Plan under the COVID-19 Epidemic}

The process of home care under the NCP epidemic includes the following steps, (1) Assessment and preparation before home care are taken including: i. Home visiting nurses call to assess the epidemiological history of NCP in the elderly and their families, ii. Home visiting nurses assess the situation of the COVID-19 epidemic in the community of the elderly, iii. Home visiting nurses assess the epidemic prevention and control measures and the traffic situation of the community where the elderly live; iv. Home visiting nurses inform the elderly and their families of the risks of home care under the COVID-19 epidemic; v. the home visiting nurses prepare all kinds of protective equipment such as quick-drying hand disinfectant, N95 mask, goggles, gloves, protective clothing, etc. (2) Home care process is carried out including: i. Home visiting nurses use quick-drying hand disinfectant for hand disinfection and wear protective equipment when they arrive at the elderly patient's home; ii. Home visiting nurses carry out health education such as cough etiquette, and hand hygiene for the elderly and their families [9], and instruct the elderly and their families to wear masks and keep the distance between people more than 1 meter [9], iii. Home visiting nurses make a comprehensive assessment of the elderly, their families and their home environment and provide home care. (3) Home visiting nurses follow up the elderly after providing home care.

\subsubsection{Developing the Contingency Plan of Home Care under the COVID-19 Epidemic}

The process of home care contingency plan under the COVID-19 epidemic includes the following steps. (1) The home visiting nurses call to assess the elderly and their families by using the COVID-19 epidemiological questionnaire which is composed of three parts including demographic information, epidemiological history and suspected symptoms of NCP.(2) The home visiting nurses need to guide the elderly and their families to seek medical advice if the elderly or their family have a history of epidemic exposure or have suspected symptoms of NCP. The home visiting nurses will provide home care if the elderly or their families have no history of exposure to the epidemic or no similar symptoms of NCP. (3) The home visiting nurses report it to their supervisor if they discover that the elderly or their families have suspected symptoms of NCP during home care period.

\subsubsection{Training Home Visiting Nurses of the Protection Knowledge and Skills of NCP}

Manager provides home visiting nurses with NCP protection knowledge training including (1) Learning about transmission, clinical manifestations, diagnosis and treatment, epidemic prevention and control and public health education of NCP, (2) Training home visiting nurses to wear protective equipment correctly, (3) Making a simulation of home care under the NCP epidemic.

\subsubsection{Paying Attention to the Physical State and Psychological State of the Home Visiting Nurses}

Manager pays attention to the physical state and psychological state of the visiting home nurses and takes a series of measures including (1) Providing adequate protective equipment for home visiting nurses, (2) Arranging shift reasonable for home visiting nurses to ensure their adequate rest time, (3) Monitoring the physical condition of home visiting nurses through daily health information declaration, (4) Encouraging home visiting nurses to discuss their feelings and offer support for them.

\subsubsection{Providing Personalized Home Care and NCP Prevention Guidance for the Elderly and Their Family}

(1) The home visiting nurse made a comprehensive assessment of the elderly, and provided home care service and health education for them; (2) The home visiting nurses assessed the nursing skills of caregivers and provided them with guidance on home care skills; (3) The home visiting nurses collected the questions of the elderly and their family about prevention of COVID-19 and conducted group health education.

\subsection{Evaluation Index}

(1) Home care cases from January 19 to March 31, 2020 are collected and compared with the same period in 2019;(2) The home visiting nurses follow up to monitor whether the elderly visit the hospital for medical treatment or adverse effect after home care;(3) Three home visiting nurses are monitored with their physical and psychological state through daily health information declaration.

\section{Results}

From January 19 to March 31, 2020, the home visiting nurses provided home care service for 93 elderly patients at home during the epidemic period, representing a $20 \%$ increase 
over the same period last year. The home care service included retention / replacement of gastric tube, retention / replacement of urinary tube, pressure ulcer care, sputum suction care, maintenance of peripherally inserted central catheters and venous blood collection for 127 times.

Through follow-up, 93 elderly at home did not have adverse effects and did not visit the hospital for medical treatment.

Three home visiting nurses were in good physical condition without any bad mood during the epidemic period.

\section{Discussion}

\subsection{Home Care Could Play a Great Role in Prevention and Control of the COVID-19 Epidemic}

Hospitals are crowded workplaces and are among the most dangerous places for cross-infection.

Chinese national government suggested that the public did not go out and visit hospitals as little as possible under the NCP epidemic. Accordingly, from January 19 to March 31,2020 , our home visiting nurses provided home care for 93 elderly patients at home during the epidemic period, representing a $20 \%$ increase over the same period last year. It showed that more home-based elderly people had home care needs under the epidemic of NCP. The national government has proposed that medical staffs should strengthen the publicity of knowledge on epidemic prevention and control for the elderly and their families, and improve their ability of prevention and control [4]. Qi $\mathrm{Y}$ et al conducted a study on the cognition, attitude and behavior of NCP among the public, and found out that the public was highly concerned about NCP and took active protective measures [10]. However, our home visiting nurses learned that the public's interpretation of the knowledge about NCP epidemic varied due to their different educational background and cognition, and most of them lacked the skills about epidemic prevention and control. Therefore, as professional medical staffs, home visiting nurses not only provided home care service for the elderly, but also carried out group health education for their family. Under the epidemic situation of NCP, home care service conform to the national policy guidance, which could not only meet the medical needs of the elderly at home, but also effectively alleviate the burden of epidemic prevention and control and reduce the risk of cross-infection in hospitals.

\subsection{Manager Should Take Measures to Ensure the Safety of Both Nurses and the Elderly under the COVID-19 Epidemic}

Due to its high infectivity, people are generally susceptible to COVID-19, especially the elderly [11]. A series of procedures and programs about prevention and control of NCP epidemic should be developed to ensure the safety of both nurses and the elderly during home care service period. Accordingly, Department manager rapidly consulted the hospital's infection control experts and formulated the process and contingency plan of home care under the COVID-19 epidemic to provide protection guidance for home visiting nurses. Besides, the manager organized home visiting nurses to conduct many trainings of NCP knowledge, and do scenario simulation of home care service procedures and contingency plans. The formulation and implementation of the above schemes have reduced the risk of infection among nurses and the elderly, and enhanced the sense of security of both sides. The 93 elderly and their families were in good condition, and the home visiting nurses were in good condition, too.

\subsection{Manager Needs to Pay More Attention to the Psychological State and Physical State of Nurses under the COVID-19 Epidemic}

Clinical nurses are under pressure both physically and psychologically during the epidemic period. Zhang WH found out that clinical nurses showed anxiety due to fear of infection under the COVID-19 epidemic [12]. Manager should be more concerned about physical state and psychological state of the home visiting nurses. According to the notice on guidelines for emergency psychological crisis intervention about NCP issued by Disease Prevention and Control Bureau of China [13], Manager developed a series of intervention including providing adequate protective equipment, arranging shift reasonable, monitoring nurses' physical states and encouraging home visiting nurses to discuss their feelings. The home visiting nurses were in good physical condition without any bad mood.

\section{Conclusion}

$95.75 \%$ of the elderly with chronic diseases have great demands of medical, nursing service and daily life care service [14]. For elderly patients, home care service can enable them to get professional nursing service in a comfortable environment. More elderly patients will have the needs of home care service under the COVID-19 epidemic. Home care could not only take advantage under the special epidemic situation, but also meet the medical needs of elderly patients at home, and effectively alleviate the burden of epidemic prevention and reduce the risk of cross infection. Home care could play a great role in prevention and control of the COVID-19 epidemic.

\section{References}

[1] Zhu HY, Li W, Niu P. The novel coronavirus outbreak in Wuhan, China [J]. Global Health Research and Policy. 2020; 5: 6. https://doi.org/10.1186/s41256-020-00135-6.

[2] Tian HY. 2019-nCoV: new challenges from coronavirus. Chin J $\begin{array}{lllll}\text { Prev } & \text { Med. 2020; } 54 & \text { (0): }\end{array}$ https://doi.org/10.3760/cma.j.issn.0253-9624.2020.0001.

[3] Song F, Shi N, Shan F, et al. Emerging coronavirus 2019-nCoV pneumonia. $\quad$ Radiology. 2020: 200274. https://doi.org/10.1148/radiol.2020200274. 
[4] Health Secretary for the Elderly of National Health Commission of China. The notice on the prevention and control of novel coronavirus pneumonia in the elderly. [2020-01-28]. http://www.nhc.gov.cn/lljks/tggg/202001/96e82ba8a14d41b28 3da990d39771493.shtml.

[5] National Health Commission of China. The national health commission held a press conference to introduce the guidelines on establishing and improving the health service system for the elderly [EB/OL]. [2020-02-08]. http://www.gov.cn/xinwen/2019-11/02/content_5448445. Html.

[6] Deng J, Jiang SZ. A study on the health needs of the aging population in China [J]. Hospital Management Forum, 2012, 29 (3): 21-24.

[7] Zhu XM, Zhai XQ. et al. Analysis on the effect of home care for elderly patients with chronic diseases [J]. Journal of Nursing Administration, 2013, (10): 726 - 728.

[8] Miao XH, Zhao LH, Tu SY, et al. Research Progress on readmission rate and related factors of stroke patients [J]. Chinese Nursing Research. 2016, 30 (5B): 1684-1690.

[9] Hirdes JP, Fries BE, Morris JN, et al. Home care quality indicators (HCQIs) based on the MDS-HC [J]. Gerontologist, 2004, 44 (5): 665-679.

[10] Qi Y, Chen LH, Zhang L, et al. Public cognition, attitude and behavior of new coronavirus infection pneumonia [J/OL].Journal of Tropical Medicine: 1-12 [2020-02-09]. https://kns-cnki-net.webvpn.jnu.edu.cn/kcms/detail/44.1503.R .20200204.1114.002.html.

[11] Medical Administration Bureau of China. The notice on guidelines for prevention and control of infection in home isolation medicine of pneumonia in the novel coronavirus pneumonia issued by National Health Commission of the People's Republic of China (Trial) [EB/OL] [2020-02-05]. http://www.nhc.gov.cn/yzygj/s7659/202002/cf80b05048584f8 da9b4a54871c44b26.shtml.

[12] Zhang $\mathrm{WH}, \mathrm{Li} \mathrm{Y}$, Zheng $\mathrm{LP}$, et al. Investigation and Countermeasures on anxiety of nurses in a designated hospital of novel coronavirus pneumonia in Hangzhou [J/OL]. Health $\begin{array}{llll}\text { Research. } & 2020 & (02): & 1-4\end{array}$ [2020-02-09]. https://kns-cnki-net.webvpn.jnu.edu.cn/kcms/detail/33.1359.R .20200205.1810.004.html.

[13] Medical Administration Bureau of China. The notice on guidelines for emergency psychological crisis intervention about novel coronavirus pneumonia [EB/OL]. [2020-01-27]. http://www.nhc.gov.cn/jkj/s3577/202001/6adc08b966594253b 2b791be5c3b9467.shtml

[14] Li JG, Dai FM, Chen RY, et al. Advances in the application of health promotion model in chronic disease service for the elderly [J]. Chinese Journal of Gerontology2019, 39 (04): 1009-1012. 